\title{
How can we Prove that Tumor Board is a Mandatory Component of High Quality Cancer Care?
}

\author{
Ahmed Nadeem Abbasi*, ${ }^{*}$, Sehrish Abrar ${ }^{1}$, Benazir Mir Khan ${ }^{2}$ \\ ${ }^{1}$ Department of Oncology, Aga Khan University, Karachi, Pakistan. \\ ${ }^{2}$ Department of Radiation Oncology, Odette Cancer Center, Sunnybrook Hospital, Toronto, Canada.
}

\section{INTRODUCTION}

Before embarking upon the first modality of cancer management, it is advisable to thoughrouly discuss the patients case in a site specific multidisciplinary tumor board meeting . These meetings are a necessary requirement for a high quality comprehensive cancer service. Published evidence reveals a positive relationship in the form of treatment outcomes. The outcomes are being measured in terms ofs of survival and local control of malignancies for patients who were discussed and deliberated nary tumour boards $[1,2]$. These boards are vitally essential for the services offered in Low and Middle Income countries as we regard them as the lifeline for our cancer patients [3].

Tumor boards provide us with an opportunity to review the quality of preliminary workup, investigations and in certain cases we do necessary changes in the tumour staging and sub-staging. The process ensures an accurate recommendation for every case discussed in the board. In developing countries, due to a variety of reasons, we have observed that it is not possible to discuss every case in a site-specific tumour board at every institute. An example of independent tumor board in Pakistan lead to the formation of City Tumor Board which is being attended by experts from all concerned specialities. This is an independent, non-institutional and non-territorial board which is being conducted on Sunday mornings at a neutral venue. This city tumour board serves to all those cancer cases who have no access to an institutional tumour board and a thorough multi-disciplinary discussion is sought. In 2013, a clinical audit of this non-institutional tumour board was being published in the Journal of the Pakistan Medical Association [4].

In the absence of a national cancer registry and a national cancer plan, we can only focus on patient-centred approaches, like the ones which improve our clinical decision making geared towards an improvement in our clinical outcomes. Development of high quality site-specific multi-disciplinary tumour boards is one of the examples of such patient centred, focused and practical endeavour. Cancer care providers serving in the developing countries rarely have direct involve-

*Address correspondence to this author at the Department of Oncology, Aga Khan University, Karachi, Pakistan.

E-mail: nadeem.abbasi@aku.edu ment in the national healthcare policy-making corridors of the country. Hence, we take the route of solving the problems faced by our patients with a head on problem solving approach. Starting a city tumour board in 2010 is an example of this strategic plan which is only addressing a portion of the wider problem [5].

Oncology clinical practice guidelines which are published regularly in developed countries are also over emphasizing on the fact that we shall form properly represented and mandated expert committees in the form of site-specific multi-disciplinary forums which oversee and monitor the site-specific tumour boards in institutions where cancer patients are managed [6].

A set of quality indicators will monitor the performance of these tumour boards via looking into the quality of recommendations made after thorough deliberations in these meetings. Day by day our learning curve is improving and we are addressing quality of these professional activities [7].

Our paient centered approach improves with a multi-disciplinary culture. A site-specific surgical team who is conducting complex oncological operative procedures cannot function without expert radiation and medical oncology teams as most malignancies are being managed by more than one modality of treatment. Tumor Board becomes imperative in certain types of malignancies, for example, locally advanced breast and rectal cancers, in which neo-adjuvant oncological treatment is recommended before the surgical removal of tumour [8]. Extensive surgical procedures like whole limb amputation, total laryngectomies and abdominoperineal resections can be avoided, if all cancer cases are discussed in multi-disciplinary site-specific tumour boards. Pathological and radiological work up and biopsy of lesions is conducted by surgical teams, as patients seek the first consultation in surgical consulting clinics. In most diagnosed cases, it becomes the responsibility of the surgical team to bring all such cases in respective site-specific boards for a thorough discussion before embarking on the surgical procedure. The frequency of these boards also plays a major role in the reluctance of bringing cases. It is recommended to perform weekly boards to ensure swift decision making and faster start of the first modality of the management plan. Leaving cases in pending does not serve the purpose and destroys the theme to 
establish these multi-disciplinary teams. In institutional practice in all site specific tumor boards a clear documentation is mandatory and proforma is being maintained.

All professional development plans in academic institutes can take an extra benefit from the establishment of these site-specific tumour boards. The cases can be prepared and presented by the postgraduate trainee students and during the process of preparation and presentation, their clinical acumen will improve [9]. Also, working in a multi-disciplinary culture, we are expecting to develop future specialists who will know their limitations. This fact applies to faculty professional growth and development. Our can incorporate the participation of concerned faculty in this activity in annual appraisal as an integral entry under clinical services heading. It is being advised to establish site-specific multi-disciplinary tumour boards in all hospitals where cancer care is provided [10].

\section{CONFLICT OF INTEREST}

Declared none.

\section{ACKNOWLEDGEMENTS}

We acknowledge all our fellow colleagues.

\section{REFERENCES}

[1] Chang JH, Vines E, Bertsch H, et al. The impact of a multidisciplinary breast cancer center on recommendations for patient management: The University of Pennsylvania experience. Cancer 2001; 91(7): 1231-7.

DOI: $\quad 10.1002 / 1097-0142(20010401) 91: 7<1231:: A I D-C N-$ CR1123>3.0.CO;2-K

[2] Kurpad R, Kim W, Rathmell WK, et al. A multidisciplinary approach to the management of urologic malignancies: Does it influence diagnostic and treatment decisions? Urol Oncol 2011; 29(4): 378-82. DOI: 10.1016/j.urolonc.2009.04.008

[3] Pellizzon C. The tumor boards - is this strategy worth for developing countries? J Contemp Brachytherapy 2018; 10: 191-2. DOI: $10.5114 /$ jcb.2018.76958

[4] Asghar AH, Abbasi AN, Jamal A, Haider G, Rizvi S. City tumour board Karachi: An innovative step in multidisciplinary consensus meeting and its two years audit. JPMA 2013; 63(12): 1534-5.

[5] Syed DS. Health: Cancer Care and Policies. The Daily Dawn. Accessed on [January 05, 2020]; Available from: https://www.dawn.com/news/1525847

[6] Network NCC. National Comprehensive Cancer Network 2020. Accessed on [January 20, 2020]; Available from: https://www.nccn.org/professionals/physician_gls/default.as$\mathrm{px}$

[7] Abbasi AN. Establishment and maintenance of quality of site-specific multidisciplinary tumor boards in Pakistan. JCPSP 2016; 26(10): 805-7.

[8] Palmer G, Martling A, Cedermark B, Holm T. Preoperative tumour staging with multidisciplinary team assessment improves the outcome in locally advanced primary rectal cancer. Colorectal Dis 2011; 13(12): 1361-9. doi: 10.5114/jcb.2018.76958 DOI: $10.5114 /$ jcb.2018.76958

[9] Mattes MD. Multidisciplinary oncology education: Going beyond tumor board. J Am Coll Radiol 2016; 13(10): 1239-41. DOI: 10.1016/j.jacr.2016.06.005

[10] A Report by the Expert Advisory Group on Cancer to the Chief Medical Officers of England and Wales. A policy framework for commissioning cancer services - the Calman-Hine Report. London, UK: Department of Health 1995. 